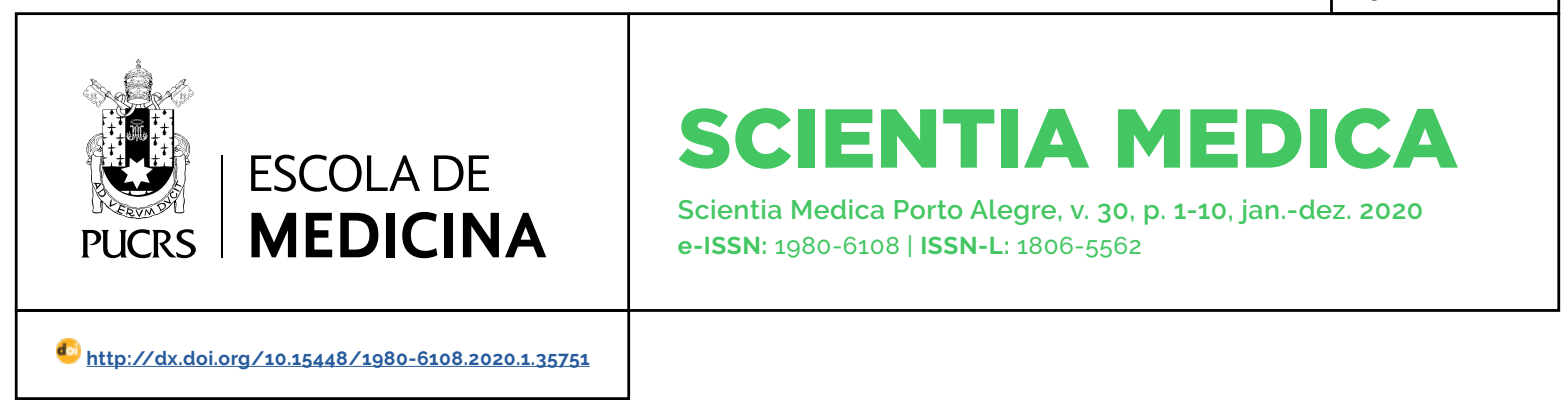

ARTIGO ORIGINAL

\title{
Internações e custos hospitalares por quedas em idosos brasileiros
}

\author{
Hospitalizations and hospital costs due to falls in Brazilian elderly
}

Filipe José da Silveira ${ }^{1}$

orcid.org/0000-0001-6041-895X

filipesilveira29@hotmail.com

\section{Vinícius da Silva Lessa de Oliveira ${ }^{1}$}

orcid.org/0000-0001-8981-0576

lessaviniciusdeoliveira@gmail.com

\section{Frederico Orlando Friedrich ${ }^{2}$}

orcid.org/0000-0001-6939-0340 fredericofriedrich@gmail.com

\section{João Paulo Heinzmann- Filho $^{1}$}

orcid.org/0000-0002-8426-0250

joaopauloheinzmann@hotmail.com

Recebido em: 2 jan. 2020. Aprovado em: 7 abr. 2020. Publicado em: 24 jul. 2020

\section{(c) (1)}

Artigo está licenciado sob forma de uma licença Creative Commons Atribuição 4.0 Internacional.

\section{Resumo}

Objetivos: verificar o número e as causas de hospitalizações por quedas em idosos brasileiros, além dos gastos federais do Sistema Único de Saúde (SUS), no periodo de 2000 a 2018.

Métodos: trata-se de um estudo ecológico, utilizando informações disponiveis na base de dados do Departamento de Informática do SUS. Foram coletados dados de idosos ( $\geq 60$ anos) que internaram no SUS devido às quedas no periodo de 2000 a 2018 no Brasil. Extraíram-se o número de hospitalizações no País e nas regiões (Norte, Nordeste, Sul, Sudeste e Centro-Oeste), as causas das quedas (Classificação Estatística Internacional de Doenças e Problemas Relacionados à Saúde -10) e o valor de gastos federais. Para fins estatísticos, utilizou-se análise descritiva.

Resultados: totalizaram-se 1,48 milhões de hospitalizações por quedas em idosos no Brasil, com uma taxa de 38,6 a cada 10 mil. As principais causas desses registros no DATASUS foram as "quedas sem especificações", as "outras quedas no mesmo nivel" e as "quedas no mesmo nivel por escorregão, tropeção ou passos em falsos". Em relação às localidades, os idosos pertencentes às regiões Sudeste $(47,1)$, Sul $(44,1)$ e Centro-Oeste $(40,4)$ foram aqueles que apresentaram maiores medianas das taxas de hospitalizações por quedas no periodo analisado. Entretanto, o Nordeste (variação\%=0,4), o Sul (variação\%=0,2) e o Centro-Oeste (variação\%=0,2) demonstraram maiores elevações desse indicador ao longo dos 18 anos, enquanto apenas a região Norte apresentou redução (variação\%=-0,5). A mediana de gastos hospitalares federais (milhões) foi de $\mathrm{R} \$ 135.58$, variando de $R \$ 112,89$ até $R \$ 194,98$.

Conclusões: houve aumento das taxas de hospitalizações por quedas em idosos no SUS em quase todas as unidades federativas. As causas mais frequentes foram as "quedas sem especificações", as "outras quedas no mesmo nivel" e as "quedas no mesmo nivel por escorregão, tropeção ou passos em falsos". Além disso, ocorreu elevação dos gastos hospitalares federais ao longo do período no País.

Palavras-chave: acidentes por quedas, indicadores básicos de saúde, gastos hospitalares, idosos, Brasil.

\section{Abstract}

Aims: to verify the number and causes of hospitalizations for falls in Brazilian elderly, in addition to the federal expenses of the Unified Health System (SUS), in the period from 2000 to 2018.

Methods: this is an ecological study, using information available in the database of the SUS Department of Informatics. Data were collected from elderly ( $\geq 60$ years) who were admitted to SUS due to falls in the period from 2000 to 2018 in Brazil. The number of hospitalizations in the country and in the regions (North, Northeast, South, Southeast and Midwest), the causes of falls (International Statistical Classification of Diseases and Related Health Problems - 10) and the amount of federal spending were extracted. For statistical purposes, descriptive analysis was used.

\footnotetext{
Centro Universitário Cenecista de Osório (UNICNEC), Curso de Fisioterapia, Osório, RS, Brasil.

Pontificia Universidade Católica do Rio Grande do Sul (PUCRS), Programa de Pós Graduação em Pediatria e Saúde da Criança, Porto Alegre, RS, Brasil.
} 
Results: there were 1.48 million hospitalizations for falls in the elderly in Brazil, with a rate of 38.6 per 10,000 . The main causes of these records in DATASUS were "falls without specifications", "other falls on the same level" and "falls on the same level due to slips, trips or false steps". Regarding the locations, the elderly belonging to the Southeast (47.1), South (44.1) and Midwest (40.4) regions were those who had the highest median hospitalization rates for falls in the analysed period. However, the Northeast (variation $\%=0.4$ ), the South (variation $\%=0.2$ ) and the Midwest (variation\%=0.2) showed greater increases in this indicator over the age of 18 , while only the North region showed a reduction (variation $\%=-0.5$ ). The median federal hospital expenses (million) was R $\$ 135.58$, ranging from $\mathrm{R} \$ 112.89$ to $\mathrm{R} \$ 194.98$

Conclusions: there was an increase in hospitalization rates due to falls in the elderly in SUS, in almost all federative units. The most frequent causes were "falls without specifications", "other falls on the same level" and "falls on the same level due to slips, trips or false steps". In addition, there was an increase in federal hospital spending over the period in the country.

Keywords: accidental falls, health status indicators, hospital expenses, elderly, Brazil.

ABREVIATURAS: ANS, Agência Nacional de Saúde Suplementar; CID-10, Classificação Estatística Internacional de Doenças e Problemas Relacionados à Saúde - $10^{a}$ revisão; DATASUS, Departamento de Informática do SUS; IBGE, Instituto Brasileiro de Geografia e Estatística; SUS, Sistema Único de Saúde.

\section{Introdução}

O envelhecimento vem ocorrendo em ordem mundial. No Brasil, acredita-se que esse processo seja devido às conquistas sociais e políticas, associadas aos avanços tecnológicos (1). De acordo com o Instituto Brasileiro de Geografia e Estatística (IBGE), o número de idosos em 2017 alcançou 33,2 milhões no País. Isso se deve às baixas taxas de mortalidade e de fecundidade, reduzindo o peso relativo da população jovem e, consequentemente, contribuindo para o envelhecimento populacional (1-3).

Aparentemente, definir o envelhecimento é algo difícil por não haver marcador biofisiológico que permita a quantificação deste fenômeno, abarcando todas as suas caracteristicas. Entretanto, pode ser entendido como um processo progressivo, irreversivel e não patológico, resultando em modificações morfofisiológicas, bioquímicas, psicológicas e funcionais. Está relacionado dinamicamente com fatores genéticos, ambientais, étnico-raciais, assim como hábitos de vida (4-7).
Com sua progressão, ocorre transição na composição corporal, como redução na osteogênese, associada ao aumento da degradação do tecido ósseo e alterações no tecido colagenoso, reduzindo a flexibilidade. Há também, diminuição de massa muscular, redução do equilíbrio e da funcionalidade, além do aumento de tecido adiposo, o que pode favorecer para a ocorrência de quedas nesse grupo etário (5-6).

As quedas são definidas como eventos que levam o indivíduo a cair de maneira não intencional, abaixo do nivel que se encontrava, gerando insegurança devido ao medo do evento acontecer novamente (6-9). Com isso, as quedas podem predispor a redução da independência funcional, além de lesões, como fratura de fêmur e traumatismo cranioencefálico, resultando em hospitalizações e gastos para o Sistema Único de Saúde (SUS) (8-11).

Estudos prévios $(12,13)$ realizados por meio do Departamento de Informática do SUS (DATASUS) reportam quase 100 mil internações por quedas em idosos em 2013 no Brasil. Também apontam gastos de aproximadamente $\mathrm{R} \$ 103$ milhões aos cofres públicos, no periodo entre 2005 e 2010 (13). Idosos geram de 2,5 a 8 vezes mais gastos hospitalares e apresentam maiores probabilidades de complicações, quando comparados aos jovens e adultos $(9,10)$. Tal fato se deve às patologias crônicas associadas com o avançar da idade, incluindo a hipertensão arterial sistêmica, o acidente vascular encefálico e a diabete melito (8).

Até o presente momento, poucas pesquisas investigaram o número de hospitalizações por quedas e seus gastos em idosos no Brasil (13-16). Além disso, esses trabalhos são limitados quanto ao período de avaliação temporal, o que justifica o desenvolvimento deste estudo $(12,13)$. Portanto, considerando a elevada proporção de idosos no País, os possiveis declínios em suas capacidades físicas e as prováveis morbidades clínicas relacionadas à idade avançada (5), o objetivo foi verificar o número e as causas de hospitalizações por quedas em idosos brasileiros, além dos gastos federais do SUS, no periodo de 2000 a 2018. 


\section{Métodos}

Trata-se de um estudo ecológico, de caráter retrospectivo. A pesquisa foi realizada por meio do DATASUS, anteriormente conhecido como Autorização de Internação Hospitalar. Utilizou-se o banco de dados DATASUS, de acesso livre do Governo Federal Brasileiro.

Foram utilizados dados de idosos ( $\geq 60$ anos) que internaram devido às quedas no SUS, no periodo de 2000 a 2018, nas regiões brasileiras (17). Para a coleta de dados, foi obtido o número de internações, as causas das quedas, além das regiões e do valor de gastos federais. A busca epidemiológica foi realizada através de dois caminhos no DATASUS, considerando que a base de dados disponibiliza links distintos de acordo com o periodo de investigação (1984 até 2007 e a partir de 2008).

$1^{\circ}$ caminho: "Acesso à informação" - "Informações de Saúde" - "Epidemiológicas e Morbidade" - "Morbidade Hospitalar do SUS" - "Causas externas, por local de internação - de 1998 a 2007" - "Abrangência geográfica: Brasil por região e unidade da federação" - "Conteúdo: Internações e Valor Total" - "Períodos Disponiveis: Jan/2000 a Dez/2007" - "Grupo de Causas: Woo-W19 Quedas" - "Faixa Etária 1: 60 a 69 anos, 70 a 79 anos e 80 anos e mais".

$2^{\circ}$ caminho: "Acesso à informação" - "Informações de Saúde" - "Epidemiológicas e Morbidade" - "Morbidade Hospitalar do SUS" - "Causas externas, por local de internação - a partir de 2008" - "Abrangência geográfica: Brasil por região e unidade da federação" - "Conteúdo: Internações e Valor Total" - "Periodos Disponiveis: Jan/2008 a Dez/2018" "Grupo de Causas: Woo-W19 Quedas" - "Faixa Etária 1: 60 a 69 anos, 70 a 79 anos e 80 anos e mais".

Os dados coletados foram analisados por região (Norte, Nordeste, Sul, Sudeste e Centro-Oeste) e investigados também quanto aos motivos das quedas que levaram à hospitalização nesse grupo etário. As categorias de quedas foram extraidas de acordo com a Classificação Estatística Internacional de Doenças e Problemas Relacionados à Saúde - 10. ' revisão (CID-10), variando de "WooQuedas no mesmo nivel envolvendo gelo e neve" até "W19- Quedas sem especificações" $(12,18)$.

Todas as informações foram extraídas e plota- das em uma planilha do Microsoft Excel ${ }^{\circledR}$, sendo revisadas pelos autores e, após, pelo pesquisador responsável para garantir a qualidade.

O número absoluto de hospitalizações foi extraido do DATASUS, sem nenhuma modificação numérica. A taxa de hospitalizações foi calculada utilizando o número de hospitalizações, como numerador e, o número de idosos, como denominador (registrados no IBGE - cadastrados com plano de saúde na Agência Nacional de Saúde Suplementar [ANS]), multiplicando-se por 10.000 habitantes (13), conforme recomendado pelo DATASUS (19).

As informações referentes aos individuos ( $\geq 60$ anos) com plano de saúde cadastrados na ANS foram utilizadas apenas para subtração do número total de idosos do IBGE. (http://www.ans.gov. br/anstabnet/cgi-bin/dh?dados/tabnet_br.def)

Foi calculada a mediana, o percentual e a variação percentual dos resultados em relação às hospitalizações e os gastos federais. A variação percentual foi realizada através da subtração dos resultados desses desfechos, entre os anos de 2018 e 2000, dividindo-se pelo primeiro valor numérico. A análise dos gastos hospitalares federais ao longo dos 18 anos foi realizada após a correlação monetária pelo o Índice Nacional de Preços ao Consumidor Amplo, com base no ano de 2018 (20).

Como o banco de dados do DATASUS é de livre acesso e de domínio público, não foi necessário obter a aprovação do Comitê de Ética em Pesquisa do Centro Universitário Cenecista de Osório.

\section{Resultados}

Foram contabilizadas 1,48 milhões de hospitalizações por quedas em idosos no Brasil, no periodo de 2000 até 2018. A tabela 1 apresenta todas as causas de hospitalizações por quedas em idosos no SUS, de acordo com o capítulo CID-10. As "quedas sem especificações" (46,0\%), as "outras quedas no mesmo nivel" (19,6\%) e as "quedas no mesmo nivel por escorregão, tropeção ou passos em falsos" (19.5\%) foram os motivos mais registrados no DATASUS ao longo dos 18 anos. 
TABELA 1 - Frequência das causas de hospitalizações por quedas em idosos no SUS, de acordo com o capítulo CID-10.

\begin{tabular}{|c|c|c|c|c|c|c|}
\hline \multirow[t]{2}{*}{ Capitulo CID-10 } & \multicolumn{2}{|c|}{$2000-2009$} & \multicolumn{2}{|c|}{$2010-2018$} & \multicolumn{2}{|c|}{ Total } \\
\hline & $\mathrm{N}$ & $\%$ & $\mathrm{~N}$ & $\%$ & $\mathrm{~N}$ & $\%$ \\
\hline Woo - Quedas no mesmo nível envolvendo gelo e neve & 15.083 & 2,5 & 7.463 & 0,8 & 22.546 & 1,5 \\
\hline $\begin{array}{l}\text { Wo1 - Quedas no mesmo nivel por escorregão, tro- } \\
\text { peção ou passos em falsos }\end{array}$ & 127.052 & 21,4 & 162.821 & 18,3 & 289.873 & 19.5 \\
\hline $\begin{array}{l}\text { Wo2 - Quedas envolvendo patins de rodas, esqui ou } \\
\text { pranchas }\end{array}$ & 1.666 & 0,3 & 3.852 & 0,4 & 5.518 & 0,4 \\
\hline $\begin{array}{l}\text { Wo3 - Outras quedas no mesmo nivel por colisão ou } \\
\text { empurrão }\end{array}$ & 2.802 & 0,5 & 10.849 & 1,2 & 13.651 & 0,9 \\
\hline $\begin{array}{l}\text { Wo4 - Quedas enquanto estava sendo carregado ou } \\
\text { apoiado }\end{array}$ & 1.989 & 0,3 & 3.561 & 0,4 & 5.550 & 0,4 \\
\hline W05 - Quedas envolvendo uma cadeira de rodas & 487 & 0,1 & 601 & 0,1 & 1.088 & 0,1 \\
\hline Wo6 - Quedas de um leito & 2.317 & 0,4 & 5.313 & 0,6 & 7.630 & 0,5 \\
\hline W07 - Quedas de uma cadeira & 1.369 & 0,2 & 2.095 & 0,2 & 3.464 & 0,2 \\
\hline Wo8 - Quedas de outro tipo de mobilia & 1.079 & 0,2 & 2.795 & 0,3 & 3.874 & 0,3 \\
\hline $\begin{array}{l}\text { Wog - Quedas envolvendo equipamento de play- } \\
\text { ground }\end{array}$ & 2.342 & 0,4 & 3.109 & 0,4 & 5.451 & 0,4 \\
\hline W10 - Quedas em escadas ou degraus & 18.068 & 3,0 & 25.871 & 2,9 & 43.939 & 3,0 \\
\hline W11 - Quedas em escadas de mão & 3.729 & 0,6 & 4.946 & 0,6 & 8.675 & 0,6 \\
\hline W12 - Quedas em andaime & 2.510 & 0,4 & 2.754 & 0.3 & 5.264 & 0,4 \\
\hline W13 - Quedas para fora de edificios ou outras estruturas & 7.543 & 1,3 & 6.263 & 0,7 & 13.806 & 0,9 \\
\hline W14 - Quedas de árvores & 3.314 & 0,6 & 1.709 & 0,2 & 5.023 & 0,3 \\
\hline W15 - Quedas de penhascos & 685 & 0,1 & 167 & 0,0 & 852 & 0,1 \\
\hline $\begin{array}{l}\text { W16 - Mergulhos na água causando outro traumatis- } \\
\text { mo que não afogamento }\end{array}$ & 355 & 0,1 & 441 & 0,1 & 796 & 0,1 \\
\hline W17 - Outras quedas de um nivel a outro & 25.222 & 4,2 & 48.585 & 5.5 & 73.807 & 5.0 \\
\hline W18 - Outras quedas no mesmo nivel & 96.298 & 16,2 & 194.034 & 21,8 & 290.332 & 19,6 \\
\hline W19 - Quedas sem especificações & 281.020 & 47,2 & 401.731 & 45,2 & 682.751 & 46,0 \\
\hline TOTAL & 594.930 & 100,0 & 888.960 & 100,0 & 1.483 .890 & 100,0 \\
\hline
\end{tabular}

CID-10 = Classificação Estatística Internacional de Doenças e Problemas Relacionados à Saúde - 10 ${ }^{a}$ revisão; N= número absoluto extraido do DATASUS (sem modificação numérica); \% = percentual equivalente ao total das causas de hospitalizações por quedas em idosos.

A tabela 2 demonstra detalhadamente o número, a taxa, a mediana e a variação percentual das hospitalizações por quedas em idosos, estratificadas de acordo com as regiões brasileiras. Do total de 1,48 milhões de hospitalizações, houve uma mediana de 68.148 internações por quedas em idosos ao ano. O número absoluto dessas oscilou de 51.193 (em 2000) até 123.775 (em 2018), correspondendo a uma taxa mediana de 38,6 a cada 10 mil idosos. As regiões Sudeste $(47,1)$, Sul $(44,1)$ e Centro-Oeste $(40,4)$ apresentaram as maiores medianas das taxas de hospitalizações 
por quedas, por 10.000 idosos, no período analisado. O Nordeste (variação percentual = 0,4), o Sul (variação percentual $=0,2$ ) e o Centro-Oeste (variação percentual = 0,2) apresentaram números crescentes dessas taxas. Por outro lado, apenas a região Norte apresentou redução (variação percentual $=-0,5$ ) desse indicador .

TABELA 2 - Número e taxa de hospitalizações por quedas (a cada 10 mil idosos) no SUS entre 2000 e 2018.

\begin{tabular}{|c|c|c|c|c|c|c|c|c|c|c|c|c|}
\hline \multirow{2}{*}{ Anos } & \multicolumn{2}{|c|}{ Norte } & \multicolumn{2}{|c|}{ Nordeste } & \multicolumn{2}{|c|}{ Sudeste } & \multicolumn{2}{|c|}{ Sul } & \multicolumn{2}{|c|}{$\begin{array}{c}\text { Centro- } \\
\text { Oeste }\end{array}$} & \multicolumn{2}{|c|}{ Brasil } \\
\hline & $\mathbf{N}$ & Taxa & $\mathrm{N}$ & Taxa & $\mathrm{N}$ & Taxa & $\mathrm{N}$ & Taxa & $\mathbf{N}$ & Taxa & $N$ & Taxa \\
\hline 2000 & 2.213 & 32,1 & 8.227 & 21,0 & 29.232 & 45.5 & 8.651 & 38.9 & 2.870 & 38,2 & 51.193 & 36,6 \\
\hline 2001 & 2.007 & 28,3 & 8.183 & 20,6 & 29.688 & 45,0 & 9.332 & 40,7 & 3.140 & 40,1 & 52.350 & 36.5 \\
\hline 2002 & 1.477 & 20,3 & 8.286 & 20.5 & 31.295 & 46.3 & 10.429 & 44,1 & 3.165 & 38,8 & 54.652 & 37,1 \\
\hline 2003 & 1.396 & 18,6 & 8.292 & 20,1 & 33.762 & 48,6 & 9.943 & 40,8 & 3.277 & 38,7 & 56.670 & 37.5 \\
\hline 2004 & 1.318 & 17,0 & 8.313 & 19,8 & 36.084 & 50,4 & 10.619 & 42,1 & 3.572 & 40,4 & 59.906 & 38,5 \\
\hline 2005 & 1.325 & 16,5 & 8.535 & 19,8 & 36.654 & 49.5 & 11.034 & 42,2 & 3.820 & 41,4 & 61.368 & 38,2 \\
\hline 2006 & 1.565 & 18,8 & 9.752 & 22,1 & 37.455 & 48,8 & 10.764 & 39,6 & 3.973 & 41,1 & 63.509 & 38,3 \\
\hline 2007 & 2.091 & 24,3 & 10.456 & 23,0 & 39.220 & 49,2 & 11.591 & 41,0 & 3.839 & 37.9 & 67.197 & 39,1 \\
\hline 2008 & 1.891 & 21,1 & 9.743 & 20,9 & 33.876 & 40,8 & 10.878 & 37,0 & 3.549 & 33,4 & 59.937 & 33.5 \\
\hline 2009 & 2.006 & 21,5 & 11.248 & 23,4 & 38.377 & 44,3 & 12.382 & 40,4 & 4.135 & 37,1 & 68.148 & 36.7 \\
\hline 2010 & 1.887 & 17,3 & 13.112 & 23,8 & 43.990 & 46,6 & 15.601 & 47,3 & 4.934 & 39.5 & 79.524 & 38,6 \\
\hline 2011 & 1.770 & 15,6 & 14.645 & 26,0 & 46.323 & 47,1 & 16.733 & 48,7 & 5.469 & 41,8 & 84.940 & 39,8 \\
\hline 2012 & 1.746 & 14,8 & 15.049 & 26,0 & 47.033 & 46,0 & 16.658 & 46.5 & 5.933 & 43,4 & 86.419 & 39,0 \\
\hline 2013 & 2.093 & 17,1 & 16.127 & 27,2 & 50.538 & 47,5 & 18.093 & 48,4 & 6.461 & 45,1 & 93.312 & 40,6 \\
\hline 2014 & 2.666 & 20,9 & 18.407 & 30,3 & 51.320 & 46,3 & 18.464 & 47,3 & 5.994 & 39.9 & 96.851 & 40,6 \\
\hline 2015 & 2.805 & 21,1 & 19.108 & 30,6 & 53.485 & 46.3 & 19.859 & 48,8 & 6.845 & 43,6 & 102.102 & 41,2 \\
\hline 2016 & 2.861 & 20,7 & 19.643 & 30,6 & 57.766 & 48,0 & 20.447 & 48,2 & 7.135 & 43,4 & 107.852 & 41,9 \\
\hline 2017 & 2.869 & 19,9 & 21.930 & 33,2 & 60.577 & 48,3 & 21.463 & 48,6 & 7.346 & 42,6 & 114.185 & 42,7 \\
\hline 2018 & 3.300 & 21,9 & 24.551 & 36,1 & 63.515 & 48,7 & 23.698 & 51,4 & 8.711 & 48,3 & 123.775 & 44,6 \\
\hline TOTAL & 39.286 & 387,8 & 253.607 & 474.9 & 820.190 & 893,2 & 276.639 & 841,9 & 94.168 & 774,6 & 1.483 .890 & 741,0 \\
\hline Mediana & 2.006 & 20,3 & 11.248 & 23,4 & 39.220 & 47,1 & 12.382 & 44,1 & 4.135 & 40,4 & 68.148 & 38,6 \\
\hline Variação \% & 0,3 & $-0,5$ & 0,7 & 0,4 & 0,5 & 0,1 & 0,6 & 0,2 & 0.7 & 0,2 & 0,6 & 0,2 \\
\hline
\end{tabular}

$\mathrm{N}$ = número absoluto extraído do DATASUS (sem modificação numérica); Taxa de hospitalizações= [número de hospitalizações / (população idosa cadastrada no IBGE - idosos registrados com plano de saúde na ANS) $x$ 10.000 habitantes]; Variação \%= variação percentual.

A tabela 3 demonstra os gastos hospitalares federais do SUS nos últimos 18 anos. A mediana de gastos foi $\mathrm{R} \$ 135,58$ milhões, variando de $\mathrm{R} \$ 112,89$ em 2000 até R\$194,98 milhões em 2018. As regiões que apresentaram maiores elevações nos gastos (em milhões) foram a Nordeste (2000: 14,70 versus 2018: 34,60; variação percentual = 0,58), a Sul (2000: 17,75 versus 2018: 39,44; variação percentual $=0,55$ ) e a Centro-Oeste (2000: 6,30 versus 2018: 10,76; variação percentual = 0,41) 
TABELA 3 - Gastos hospitalares federais, em Reais, registrados no DATASUS, entre 2000 e $2018^{*}$.

\begin{tabular}{|c|c|c|c|c|c|c|c|c|c|c|c|c|}
\hline \multirow{2}{*}{ Anos } & \multicolumn{2}{|c|}{ Norte } & \multicolumn{2}{|c|}{ Nordeste } & \multicolumn{2}{|c|}{ Sudeste } & \multicolumn{2}{|c|}{ Sul } & \multicolumn{2}{|c|}{ Centro-Oeste } & \multicolumn{2}{|c|}{ Brasil } \\
\hline & Milhões & $\%$ & Milhões & $\%$ & Milhões & $\%$ & Milhões & $\%$ & Milhões & $\%$ & Milhões & $\%$ \\
\hline 2000 & 3.39 & 7,2 & 14,70 & 3.5 & 70,76 & 4.3 & 17,75 & 3,2 & 6,30 & 4,1 & 112,89 & 4,0 \\
\hline 2001 & 3,03 & 6,4 & 15,03 & 3.5 & 67,61 & 4,1 & 18,23 & 3.3 & 6,42 & 4,2 & 110,32 & 4,0 \\
\hline 2002 & 1,90 & 4,0 & 15,29 & 3,6 & 69,68 & 4,2 & 22,64 & 4,1 & 6,34 & 4,2 & 109,51 & 3.9 \\
\hline 2003 & 1,48 & 3,1 & 15,63 & 3.7 & 76,51 & 4,6 & 21,14 & 3.9 & 6,58 & 4,3 & 121,33 & 4,4 \\
\hline 2004 & 1,40 & 3,0 & 16,47 & 3.9 & 82,75 & 5,0 & 22,63 & 4,1 & 7,54 & 5,0 & 130,79 & 4,7 \\
\hline 2005 & 1,60 & 3,4 & 17,46 & 4,1 & 81,37 & 4,9 & 23,28 & 4.3 & 7,64 & 5.0 & 131,35 & 4,7 \\
\hline 2006 & 1,92 & 4,1 & 18,38 & 4,3 & 80,88 & 4,9 & 21,88 & 4,0 & 7,22 & 4,8 & 130,28 & 4,7 \\
\hline 2007 & 2,59 & 5.5 & 19.50 & 4,6 & 82,75 & 5,0 & 23.56 & 4.3 & 7,18 & 4.7 & 135.58 & 4.9 \\
\hline 2008 & 2,66 & 5,6 & 19,42 & 4,6 & 74,72 & 4.5 & 22,44 & 4,1 & 6.53 & 4.3 & 125,78 & 4.5 \\
\hline 2009 & 3,00 & 6,4 & 22,68 & 5.3 & 86,47 & 5,2 & 25.57 & 4.7 & 7,50 & 4.9 & 145,22 & 5,2 \\
\hline 2010 & 2,59 & 5.5 & 24,23 & 5.7 & 95.75 & 5,8 & 33,00 & 6,0 & 8,77 & 5,8 & 131,34 & 4,7 \\
\hline 2011 & 2,23 & 4.7 & 24.94 & 5.9 & 94,14 & 5.7 & 35,33 & 6,4 & 8,94 & 5.9 & 165.58 & 5.9 \\
\hline 2012 & 2,03 & 4.3 & 25,36 & 6,0 & 91,97 & 5,6 & 33.34 & 6,1 & 9,38 & 6,2 & 162,08 & 5,8 \\
\hline 2013 & 2,42 & 5,1 & 26.97 & 6,3 & 98,41 & 5.9 & 37.96 & 6.9 & 9,92 & 6.5 & 175,67 & 6,3 \\
\hline 2014 & 2,93 & 6,2 & 29,83 & 7,0 & 99,41 & 6,0 & 38,16 & 7,0 & 8,19 & 5,4 & 178,53 & 6,4 \\
\hline 2015 & 2,94 & 6,2 & 27,42 & 6,4 & 94.55 & 5.7 & 37.90 & 6.9 & 8,70 & 5.7 & 171,50 & 6,1 \\
\hline 2016 & 2,92 & 6,2 & 27,28 & 6,4 & 99,97 & 6,0 & 37.57 & 6.9 & 9,03 & 5.9 & 176,77 & 6,3 \\
\hline 2017 & 2,77 & 5.9 & 30,55 & 7,2 & 103,88 & 6.3 & 36,45 & 6,7 & 9,14 & 6,0 & 182,79 & 6,6 \\
\hline 2018 & 3,43 & 7.3 & 34,60 & 8,1 & 106,76 & 6,4 & 39,44 & 7,2 & 10,76 & 7,1 & 194.98 & 7,0 \\
\hline Total & 47,23 & 100,0 & 425,74 & 100,0 & 1658,34 & 100,0 & 548,27 & 100,0 & 152,08 & 100,0 & 2792,29 & 100,0 \\
\hline Mediana & 2,59 & 5.5 & 22,68 & 5.3 & 86,47 & 5,2 & 25.57 & 4.7 & 7,64 & 5,0 & 135.58 & 4.9 \\
\hline Variação \% & \multicolumn{2}{|c|}{0,01} & \multicolumn{2}{|c|}{0,58} & \multicolumn{2}{|c|}{0,34} & \multicolumn{2}{|c|}{0,55} & \multicolumn{2}{|c|}{0,41} & \multicolumn{2}{|c|}{0,42} \\
\hline
\end{tabular}

*Valores em reais ajustados de acordo com o Índice Nacional de Preços ao Consumidor Amplo, com base no ano de 2018; Variação \%= variação percentual.

\section{Discussão}

As quedas causam redução na funcionalidade $(6,12)$, podendo desencadear fraturas, imobilidade no leito e complicações respiratórias, acarretando maior permanência hospitalar e despesas com o tratamento (21). Baseado nos resultados encontrados, 1,48 milhões de idosos hospitalizaram por quedas, correspondendo a 38 idosos a cada 10 mil sujeitos ( $\geq 60$ anos). Isso está próximo aos resultados de Abreu et al. (12), o qual obtiveram cerca de 1 milhão de internações registradas (1996 a 2012) no DATASUS. Em contraponto, encontra-se acima dos valores reportados (79.524) em outro estudo (13), no periodo entre 2005 e 2010. Essas diferenças podem ser atribuidas aos distintos periodos analisados, além de que, a cada ano, mais unidades de saúde abastecem essas informações nessa plataforma.

Até o presente momento, não foi localizado nenhum estudo que tenha divulgado a taxa de 
hospitalizações por quedas em idosos, tanto em âmbito nacional quanto internacional, inviabilizando nossa inferência. Contudo, Mahoney et al. (22) investigaram as quedas em idosos durante três meses após a hospitalização, relatando uma taxa de queda de 8 e 2,5 a cada mil pacientes por dia, nas 2 primeiras e 8 semanas após a alta, respectivamente (22). Outra pesquisa recente identificou um aumento da variação anual da taxa de mortalidade por quedas (1997: 6,8 versus 2010: 23,6) na população de idosos de Santa Catarina (23), estando relacionado ao avançar da idade cronológica e por essa região possuir elevado indice de envelhecimento populacional (24).

As regiões que apresentaram maiores números absolutos de hospitalizações por quedas foram a Sudeste, seguidas da Sul e da Nordeste, o que corrobora com os resultados de Barros et al. (13). No entanto, após a correção pelo número total da população geriátrica, as regiões com maiores taxas foram a Sudeste, a Sul e a Centro-Oeste. Uma das hipóteses que pode ser atribuida é o maior número de idosos nessas duas primeiras localidades (24), o que de certo modo, aumenta a proporção de tal evento ocorrer (25). Igualmente, vale ressaltar que essas três regiões apresentam maiores números de idosos longevos ( $\geq 80$ anos) quando comparadas às demais localidades (24). Sabe-se que idosos longevos se tornam mais vulneráveis às quedas, devido à redução de suas capacidades funcionais (26). O estudo de Cruz et al. (26) identificou uma associação significativa entre a idade (71 e 80 anos) e as quedas, indicando que quanto maior a idade, maior a predisposição para quedas. Esses achados denotam a importância da criação de medidas que favoreçam a prevenção de quedas nessa população, por exemplo, a Estratégia Brasil Amigo da Pessoa Idosa (27, 28).

No presente estudo, a região Nordeste foi a localidade com maior elevação das taxas de hospitalizações no periodo analisado. Tal acontecimento pode ser justificado pela maior taxa de analfabetismo dessa unidade no País, mesmo havendo redução de 14.5\% no ano de 2017 para 13.9\% em 2018 (29, 30). Além disso, destaca-se por apresentar menor pontuação no índice de Desenvolvimento Humano Municipal (31). Surpreendentemente, as regiões Sul e Centro-Oeste também apresentaram maiores elevações, mesmo possuindo menores taxas de analfabetismo e um dos melhores desempenhos no Índice de Desenvolvimento Humano Municipal (30, 31). Novamente, o elevado número de idosos com mais de 80 anos pode ser atribuido, pelo menos em parte, a esses achados (24). Em contrapartida, a região Norte foi a única localidade que apresentou redução desse indicador epidemiológico. Siqueira et al. (32) entendem que essa redução ocorra devido aos idosos serem mais jovens nessa área e pelas menores taxas de analfabetos (33).

Há um consenso na literatura a respeito de fatores predisponentes para as quedas em idosos, incluindo o gênero feminino, a idade avançada ( $\geq 80$ anos), a alteração na marcha e o declínio da função cognitiva $(12,32,36)$. Ao mesmo tempo, a má iluminação, o piso escorregadio e o ambiente domiciliar estão comumente associados (12, 32 , 36), o que corrobora com uma das categorias mais frequentes registradas no DATASUS, as "quedas no mesmo nivel por escorregão, tropeção ou passos em falsos". No entanto, torna-se limitada nossa inflexão quanto aos outros dois motivos de quedas, devido a plataforma não informar detalhes quanto às "quedas sem especificação" e às "outras quedas no mesmo nivel".

No Brasil, houve uma mediana em torno de R\$135.58 milhões de gastos com hospitalizações por quedas em idosos, obtendo-se um aumento nas despesas de aproximadamente R\$82,09 milhões no periodo analisado. Dentre as regiões que apresentaram maiores tarifas, destacam-se a Sudeste, a Sul e a Nordeste. Barros et al. (13) estão de acordo com nossos achados, apontando que essas localidades apresentam mais comorbidades, o que induz a mais gastos públicos. Existem relatos de que as regiões brasileiras estão passando por transições epidemiológicas desiguais, ocorrendo mais internações por doenças circulatórias, respiratórias e parasitárias na região Nordeste, e, por enfermidades cardiorrespiratórias e neoplásicas, nas regiões Sul e Sudeste (37). 
A ausência de estratificação das quedas em relação ao gênero se caracteriza como a principal limitação deste estudo. No entanto, como inúmeras pesquisas relatam claramente um predomínio de quedas em idosas (32, 38), não foi analisado esse fator. Além disso, o uso de uma plataforma manuseada por vários profissionais pode ser um viés de mensuração (35). Porém, diversos estudos (9, 10, $12,13)$ com diferentes faixas etárias vêm utilizando esses indicadores epidemiológicos (35), o que assegura os resultados encontrados.

Em conclusão, os achados evidenciaram um aumento das taxas de hospitalizações por quedas em idosos no SUS, em quase todas as unidades federativas, o que totalizou 1,48 milhões desse desfecho no Brasil. As "quedas sem especificações", as "outras quedas no mesmo nivel" e as "quedas no mesmo nivel por escorregão, tropeção ou passos em falsos" foram os motivos mais frequentes. Além disso, ocorreu elevação dos gastos hospitalares federais ao longo do período no País. Com isso, faz-se necessário revisar as medidas primárias de saúde do idoso, com ênfase em abordagens interdisciplinares, considerando todos os aspectos físicos, psicológicos e sociais envolvidos no contexto das quedas.

\section{Notas}

\section{Apoio financeiro}

Este estudo não recebeu apoio financeiro de fontes externas.

\section{Declaração de conflito de interesses}

Os autores declaram não haver conflitos de interesses relevantes ao conteúdo deste estudo.

\section{Contribuições dos autores}

Todos os autores fizeram contribuições substanciais para concepção, ou delineamento, ou aquisição, ou análise ou interpretação de dados; e redação do trabalho ou revisão crítica; e aprovação final da versão para publicação.

\section{Disponibilidade dos dados e responsabilidade pelos resultados}

Todos os autores declaram ter tido total acesso aos dados obtidos e assumem completa responsabilidade pela integridade destes resultados.

\section{Referências}

1. Lazarus NR, Harridge SDR. The inherent human aging process and the facilitating role of exercise. Front Physiol. 2018;9(1135):1-8. https://doi.org/10.3389/ fphys.2018.01135

2. Rodwell GEJ, Sonu R, Zahn JM, Lund J, Wilhelmy J, Wang L, Xiao W, Mindrinos M, Crane E, Segal E, Myers BD, Brooks JD, Davis RW, Higgins J, Owen AB, Kim SK. A Transcriptional profile of aging in the human kidney.. PLoS Biol 2(12): e427. https://doi.org/10.1371/journal.pbio.0020427

3. Brasil. Instituto Brasileiro de Geografia e Estatística (IBGE).Pesquisa Nacional por Amostra de Domicilios Contínua (PNAD Continua). Número de idosos cresce 18\% em 5 anos e ultrapassa 30 milhões em 2017. [Internet]. Rio de Janeiro; 2018. [updated 2018 Oct; cited 2018 April 26]. Available from: https://agenciadenoticias.ibge.gov.br/ agencia-noticias/2012-agencia-de-noticias/noticias 120980-numero-de-idosos-cresce-18-em-5-anos -e-ultrapassa-30-milhoes-em-2017

4. Christensen K, Doblhammer G, Rau R, Vaupel JW. Ageing populations: the challenges ahead. Lancet. 2009 3:374(9696):1196-208. https://doi.org/10.1016/ S0140-6736(09)61460-4

5. Crimmins EM. Trends in the health of the elderly. Annu Rev Public Health. 2004:25:79-98. https://doi. org/10.1146/annurev.publhealth.25.102802.124401

6. Parker MG, Thorslund M. Health trends in the elderly population: getting better and getting worse. Gerontologist. 2007:47:150. https://doi.org/10.1093/geront/47.2.150

7. Flaatten H, Skaar E, Joynt GM. Understanding cardiovascular physiology of ageing. Intensive Care Med. 2018;44(6):9325. https://doi.org/10.1007/s00134-018-5119-7

8. Motta CCR, Hansel CG, Silva J. Perfil de internações de pessoas idosas em um hospital público. Rev Eletr Enf. 2010;12(3):471-7. https://doi.org/10.5216/ree.v12i3.6865

9. Silveira RE, Santos AS, Sousa MC, Monteiro TS. Gastos relacionados a hospitalizações de idosos no brasil: perspectivas de uma década. Einstein. 2013; 11(4):514-20. https://doi.org/10.1590/S1679-45082013000400019

10. Gois ALB, Veras RP. Informações sobre a morbidade hospitalar em idosos nas internações do Sistema Único de Saúde do Brasil. Ciênc Saúde Coletiva. 2010;15(6):285969. https://doi.org/10.1590/S1413-81232010000600023

11. Ozturk TC, Rohat AK, Akoglu EU, Onur O, Eroglu S, Saritemur M. Factors associated with multiple falls among elderly patients admitted to emergency department IJGE. 2017; 11(2): 85-9. https://doi.org/10.1016/i.ijge.2016.05.009

12. Abreu DROM, Novaes ES, Oliveira RR, Mathias TAF, Marcon SS. Internação e mortalidade por quedas em idosos no Brasil: análise de tendência. Ciênc Saúde Coletiva. 2018; 23(4): 1131-41. https://doi.org/10.1590/141381232018234.09962016

13. Barros IFO, Pereira MB, Weiller TH, Anversa ETR. Internações hospitalares por quedas em idosos brasileiros e os custos correspondentes no âmbito do Sistema Único de Saúde. Rev Kairós Gerontol. 2015;18(4):63-80. 
14. Brutti B, Flores J, Hermes J, Martelli G, Porto DdeS, Anversa ETR. Diabete Mellitus: definição, diagnóstico, tratamento e mortalidade no Brasil, Rio Grande do Sul e Santa Maria, no periodo de 2010 a 2014. Braz J Hea Rev. 2019;4(2):3174-82. https://doi.org/10.34119/bjhrv2n4-083

15. Stelmach R, Cruz ÁA. O paradoxo da asma: negligência, fardo e big data. J Bras Pneumol. 2017; 43(3):159-60. https://doi.org/10.1590/s1806-37562017000300002

16. Luzardo AR, Junior NFP, Medeiros M, Wolkers PCB, Santos SMA. Repercussões da hospitalização por queda de idosos: cuidado e prevenção em saúde. Rev Bras Enferm. 2018:71(2):816-22. https://doi.org/10.1590/00347167-2017-0069

17. Brasil. Ministério da Saúde. Estatuto do Idoso. $2^{\mathrm{a}}$ ed. Brasil: Editora do Ministério da Saúde; 2009. p. 1-70.

18. Brasil. Ministério da Saúde. Informações em Saúde (TABNET). Portal da Saúde SUS. DATASUS. Base de dados do Sistema de Informações Hospitalares do SUS - SIH/SUS [Internet]. Rio de Janeiro; 2016. lupdated 2016 Apr; cited 2016 April 26]. Available from: http://tabnet.datasus.gov.br/cgi/deftohtm.exe?sih/cnv/fiuf.def

19. Brasil. Ministério da Saúde. Rede Interagencial de Informações para a Saúde (RIPSA). IDB 2010 Brasil. Indicadores de Mortalidade. D.29 Taxa de internação hospitalar (SUS) por causas selecionadas. Sistema de Informações Hospitalares do SUS - SIH/SUS [Internet]. Brasilia; 2012. [updated 2012]. Available from: http://tabnet.datasus.gov.br/cgi/deftohtm.exe?idb2010/d29.def

20. Brasil. Pesquisa Nacional por Amostra de Domicilios Contínua. Instituto Brasileiro de Geografia e Estatistica (IBGE). Índice Nacional de Preços ao Consumidor Amplo (IPCA). [Internet]. Rio de Janeiro; 2020. [updated 2020 Jan; cited 2020 Jan]. Available from: https://wwwibge.gov.br/estatisticas/economicas/ precos-e-custos/9256-indice-nacional-de-precos -ao-consumidor-amplo.html?t=series-historicas \&utm_source=landing\&utm_medium=explica\&utm campaign=inflacao\#plano-real-mes

21. Jahana KO, Diogo MJDE. Quedas em idosos: principais causas e consequências. Saúde Coletiva. 2007; 4(17):148-53.

22. Mahoney JE, Palta M, Johnson J, Jalaluddin M, Gray S, Park S, Sager M. Temporal association between hospitalization and rate of falls after discharge. Arch Intern Med. 2000;60(18):2788-95. https://doi.org/10.1001/ archinte.160.18.2788

23. Antes DL, Schneider IJC, d'Orsi E. Mortalidade por queda em idosos: estudo de série temporal. Rev Bras Geriatr Gerontol. 2015;18(4):769-78. https://doi. org/10.1590/1809-9823.2015.14202

24. Brasil. Ministério da Saúde. Instituto Brasileiro de Geografia e Estatística (IBGE). Sinopse dos Resultados do Censo 2010 [Internet]. Rio de Janeiro; 2010. lupdated 2010; cited 2018 April 03]. Available from: https://WWW. ibge.gov.br/cens02010.
25. Zhang J, Kay F. What's the Relative Risk? A Method Correcting the Odds Ratio in Cohort Studies of Common Outcomes. JAMA.1998;19(280):1690-1. https://doi. org/10.1001/jama.280.19.1690

26. Cruz DT, Cruz FM, Chaoubah A, Leite ICG. Fatores associados a quedas recorrentes em uma coorte de idosos. Cad Saúde Colet. 2017:25(4):475-82. https://doi. org/10.1590/1414-462×201700040081

27. Organização Mundial de Saúde (OMS). Organização Pan-Americana de Saúde (OPAS). OPAS/OMS Brasil. Brasil lança estratégia para melhorar vida de idosos com base em recomendações da OMS. [Internet]. Brasília; 2018. [updated 2018 Apr; cited 2018 April 03]. Available from: https://www.paho.org/bra/index.php?option= com_content\&view $=$ article\&id $=5625$ : brasil- lancaestrategia-para-melhorar-vida-de-idosos-combase-em-recomendacoes-da-oms\&ltemid $=820$

28. Pimentel WRT, Pagotto V, Stopa SR, Hoffmann MCCL, Andrade FB, Junior PRBS, Lima-Costa MF, Menezes RL. Quedas entre idosos brasileiros residentes em áreas urbanas: ELSI-Brasil. Rev Saúde Pública. 2018:52 (Supl 2:12). https://doi.org/10.11606/s15188787.2018052000635

29. Brasil. Pesquisa Nacional por Amostra de Domicilios Continua (PNAD Contínua). Instituto Brasileiro de Geografia e Estatística (IBGE). Analfabetismo cai em 2017, mas segue acima da meta para 2015. [Internet]. Rio de Janeiro; 2018. [updated 2018 May; cited 2018 May 18]. Available from: https://agenciadenoticias.ibge.gov.br/ agencia-noticias/2012-agencia-de-noticias/noticias/ 21255-analfabetismo-cai-em-2017-mas-segueacima-da-meta-para-2015

30. Peres MAC. Velhice e analfabetismo, uma relação paradoxal: a exclusão educacional em contextos rurais da região Nordeste. Soc Estado. 2011;26(3):631-62. https://doi.org/10.1590/S0102-69922011000300011

31. Soares C, Teixeira JR. O desenvolvimento socioeconômico no Nordeste e Sudeste Rev Econ. 2010;12(2):127-55.

32. Siqueira FV, Facchini LA, Silveira DS, Piccini RX, Tomasi E, Thume E, Silva E, Manjourany S, Alitéia D. Prevalence of falls in elderly in Brazil: a countrywide analysis. Cad Saúde Pública 2011; 27: 1819-26. https:// doi.org/10.1590/S0102-311X2011000900015

33. Guibu IA, Moraes JC, Junior AAG, Costa EA, Acurcio FA, Costa KS, Margô Gomes de Oliveira Karnikowski MGO, Soeiro OM, Leite SN, Álvares J. Características principais dos usuários dos serviços de atenção primária à saúde no Brasil. Rev Saúde Pública. 2017:51 (Supl 2:17). https://doi.org/10.11606/S1518-8787.2017051007070

35. Paiva RFPS, Souza MFP. Associação entre condições socioeconômicas, sanitárias e de atenção básica e a morbidade hospitalar por doenças de veiculação hídrica no Brasil. Cad Saúde Públ. 2018:34(1):01-11. https://doi. org/10.1590/0102-311×00017316

36. Gawryszewski VP. A importância das quedas no mesmo nível entre idosos no estado de São Paulo. Rev Assoc Med Bras. 2010;56(2): 162-7. https://doi. org/10.1590/S0104-42302010000200013 
37. Barbosa TC, Moro JS, Junior JNR, Yanes CY, Ribeiro ER. Causas de internações hospitalares em idosos por regiões do Brasil: Série histórica de 10 anos. Rev Saúde Públ 2019;2(1):70-81. https://doi.org/10.32811/ 25954482-2019v2supl1p70

38. Nascimento JS, Tavares DMS. Prevalência e fatores associados a quedas em idosos. Texto Contexto Enferm. 2016;25(2):e0360015. https://doi.org/10.1590/010407072016000360015

Filipe José da Silveira

Fisioterapeuta, graduando pelo Centro Universitário Cenecista de Osório (UNICNEC, Osório, RS, Brasil).

\section{Vinícius da Silva Lessa de Oliveira}

Fisioterapeuta, graduando pelo Centro Universitário Cenecista de Osório (UNICNEC, Osório, RS, Brasil).

\section{Frederico Orlando Friedrich}

Fisioterapeuta, graduando pela Universidade Regional Integrada do Alto Uruguai e das Missões (URI, Erechim, RS, Brasil), aluno do Programa de Pós-graduação em Pediatria e Saúde da Criança e pesquisador do Laboratório de Fisiologia Respiratória - Centro Infant da Escola de Medicina da Pontifícia Universidade Católica do Rio Grande do Sul (PUCRS, Porto Alegre, RS, Brasil).

\section{João Paulo Heinzmann Filho}

Fisioterapeuta, graduado pelo Instituto Cenecista de Ensino Superior de Santo Ângelo (IESA, Santo Ângelo, RS, Brasil), Mestre e Doutor em Saúde da Criança pela Pontifícia Universidade Católica do Rio Grande do Sul (PUCRS, Porto Alegre, RS, Brasil). Professor titular do Curso de Fisioterapia do Centro Universitário Cenecista de Osório (UNICNEC, Osório, RS, Brasil).

\section{Endereço para correspondência}

João Paulo Heinzmann Filho

Centro Universitário Cenecista de Osório

Rua 24 de maio, 141

95520000

Osório, RS, Brasil 